Review

\title{
Stem Cell Treatment for Alzheimer's Disease
}

\section{Ming Li ${ }^{1}$, Kequan Guo ${ }^{2}$ and Susumu Ikehara ${ }^{1, *}$}

1 Department of Stem Cell Disorders, Kansai Medical University, 2-5-1 Shinmachi, Hirakata City, Osaka 573-1010, Japan; E-Mail: liming@hirakata.kmu.ac.jp

2 Department of Cardiac Surgery, Beijing Institute of Heart, Lung \& Blood Vessel Disease, Beijing Anzhen Hospital Affiliated to Capital Medical University, Beijing 100029, China; E-Mail: guokequan@hotmail.com

* Author to whom correspondence should be addressed; E-Mail: ikehara@hirakata.kmu.ac.jp; Tel.: +81-72-804-2450; Fax: +81-72-804-2454.

External Editor: Katalin Prokai-Tatrai

Received: 6 September 2014; in revised form: 9 October 2014 / Accepted: 10 October 2014 / Published: 23 October 2014

\begin{abstract}
Alzheimer's disease (AD) is a progressive and neurodegenerative disorder that induces dementia in older people. It was first reported in 1907 by Alois Alzheimer, who characterized the disease as causing memory loss and cognitive impairment. Pathologic characteristics of $\mathrm{AD}$ are $\beta$-amyloid plaques, neurofibrillary tangles and neurodegeneration. Current therapies only target the relief of symptoms using various drugs, and do not cure the disease. Recently, stem cell therapy has been shown to be a potential approach to various diseases, including neurodegenerative disorders, and in this review, we focus on stem cell therapies for $\mathrm{AD}$.
\end{abstract}

Keywords: Alzheimer's disease; stem cell therapy; oxidative stress; neurodegeneration

\section{Introduction}

Alzheimer's disease (AD) is the most common form of dementia, which is one of the major causes of disability and dependency among older people worldwide. AD manifests as an impaired ability to comprehend or use words, poor coordination and gait, and impaired executive functions in the realms of planning, ordering and making judgments. Generally, classification of AD includes familial and sporadic AD. Familial AD presents mainly as the mutation of three genes: The amyloid precursor protein (APP), 
presenilin-1 (PS-1) and presenilin-2 [1]. Sporadic AD results from environmental factors and risk genes, with apolipoprotein (ApoE) reportedly the most important [2]. Pathologic characteristics of AD are $\beta$-amyloid (A $\beta$ ) plaques, neurofibrillary tangles (NFT) and neurodegeneration. A $\beta$ peptide is the main constituent of senile plaques, and $A \beta$ fibrils from pores in neurons have been shown to lead to calcium influx and neuronal death [3]. NFT consists of neurofibrillary protein aggregates, formed as abnormal hyperphosphorylation of tau protein, which is one of the microtubule-associated proteins [4]. These pathological changes lead to synaptic loss, neuronal dysfunction and death. Furthermore, microglia have been reported to play an important role in the immune defense system of the central nervous system (CNS). Microglia activation and the release of associated inflammatory factors has been reported to contribute to chronic neurodegenerative disorders in AD [5]. Decreasing amyloid deposits and the use of antioxidant therapies have some ability to alleviate AD and, more recently, cell therapy has been seen as a potential approach to its treatment. In this review, we focus on stem cell therapies for AD.

\section{Pathophysiology of AD}

The pathophysiology of $\mathrm{AD}$ includes loss of neurons and synapses in the cerebral cortex and parts of the subcortical areas [6]. APP is cleaved by $\alpha$-, $\beta$ - and $\gamma$-secretases, generating a soluble peptide in normal people, but APP generates A $\beta$ through abnormal processing, such as in AD. A $\beta$ oligomers are thought to be the most toxic due to their impairment of synaptic and neuronal functions, leading to neurodegeneration that is clinically manifested by memory and cognitive dysfunction [7]. Tau is a neuronal microtubule-associated protein, separated from microtubules when it is phosphorylated. It forms helical filaments and aggregates neurofibrillary tangles in the neuronal cytoplasm [8]. One report has indicated that $A \beta$ may accelerate tau aggregation into NFT, and that tau reduction can block $A \beta$ - and excitotoxin-induced neuronal dysfunction in the AD mouse model [9].

Chronic neuroinflammation is one of the major factors in the pathophysiology of AD [10], this disease being associated with an inflammatory response as a result of increasing numbers of activated microglia and astrocytes, and activated complement proteins, cytokines, and reactive oxygen [11]. Microglia may release inflammatory cytokines, chemokines, and growth factors to remedy neuronal injury, and while microglia remain in a resting state in the healthy adult brain, they undergo dramatic changes in morphology in response to injury $[12,13]$. Aged microglia show a higher level of activation, and release inflammatory cytokines such as IL-1, IL-6 and TNF $\alpha$, which are associated with age-related cortical atrophy in humans [14]. Recent research suggests that astrocytes are not simply passive support cells for neurons, but are active participants in neural information processing in the brain. TGF- $\beta$ was detected around and within senile plaques in brains from Alzheimer patients, indicating that $A \beta$ deposits may also induce an anti-inflammatory effect. Moreover, astrocytes produce IL-6, resulting in a localized inflammatory state within the CNS, and anti-inflammatory drugs such as tenilsetam show beneficial effects in AD animal models, suggesting that anti-inflammation therapy may help prevent AD [10].

Oxidative stress is a sign of aging and an important pathogenic factor in AD. Oxygen metabolism generates free radicals such as hydroxylradical, superoxide radical, and reactive nitrogen species, inducing reactive oxygen species (ROS) [15]. An imbalance between oxidant and antioxidant agents could generate oxidative stress, which induces damage to macromolecules and disrupts the reduction/oxidation (redox) signaling [16]. Mitochondria contain many redox enzymes, and generate ROS as a result of inefficient oxidative phosphorylation. Mitochondrial dysfunction occurs early and 
has a primary role in the pathogenesis of $\mathrm{AD}$ [17]. Moreover, one report has shown that the transition metals such as $\mathrm{Cu}^{2+}, \mathrm{Zn}^{2+}$ and $\mathrm{Fe}^{3+}$ are associated with $\mathrm{A} \beta$ aggregation and oxidative damage in the $\mathrm{AD}$ brain, and that $\mathrm{A} \beta$ catalyses the reduction of $\mathrm{Cu}^{2+}, \mathrm{Fe}^{3+}$ and $\mathrm{H}_{2} \mathrm{O}_{2}$, leading to the formation of proapoptotic lipid peroxidation products [18]. A $\beta$, which accumulates in parenchyma and blood vessels causes microglial migration and promotes inflammatory responses. A $\beta$ also decreases ATP and increases ROS generation in mitochondria, leading to cell apoptosis $[19,20]$.

\section{General Treatment for AD}

Deposits of $A \beta$ are a pathological hallmark of $A D$, and thus depleting $A \beta$ should be a useful therapy for $\mathrm{AD}$. Cathepsin $\mathrm{B}$ is a cysteine protease of the papain superfamily, and degrades peptides and proteins that enter the endolysosomal system by endocytosis or phagocytosis [21]. Extracellular Cathepsin B is associated with amyloid plaques, and colocalizes with $A \beta$ in regulated secretory vesicles in chromaffin cells in $\mathrm{AD}$ brains [22]. One report demonstrated that Cathepsin $\mathrm{B}$ reduces the relative abundance of $\mathrm{A} \beta$ through limited proteolysis, suggesting that the activation of Cathepsin $B$ could offer a therapeutic strategy for $\mathrm{AD}$ [23]. Neprilysin is another major extracellular $\mathrm{A} \beta$ degrading enzyme that clears $\mathrm{A} \beta$ from the brain, and the injection of human neprilysin decreased amyloid plaques in the transgenic mouse [24]. AD therapeutic options also include acetylcholinesterase inhibitors and the $N$-methyl-D-aspartate receptor antagonist memantine, a glutamate receptor associated channel blocker, although there are side effects in the treatment of $\mathrm{AD}[25]$.

Glutathione is an antioxidant in brain cells. It reacts with ROS and oxidized products forming gluthathione disulphide. Vitamin $\mathrm{E}$ is another endogenous antioxidant that protects against lipid peroxidation, and high levels of vitamin $\mathrm{E}$ have been shown to be related to a reduced risk of $\mathrm{AD}$ [26]. Vitamin $\mathrm{C}$ is a water-soluble antioxidant that is necessary for the reactivation of vitamin $\mathrm{E}$. Although vitamins $\mathrm{C}$ and $\mathrm{E}$ have been used in clinical applications to prevent $\mathrm{AD}$, no clear beneficial effect has been demonstrated [2].

\section{Stem Cell Treatment for AD}

Stem cells include embryonic stem cells (ESCs), induced pluripotent stem cells (iPSCs), and tissue-derived stem cells, such as bone marrow (BM)- and adipose-derived stem cells. Stem cell-derived neurons have the potential to integrate into the existing neural networks of the host brain [27]. And stem cell transplantation appears to increase acetylcholine levels to improve cognition and memory in the animal model [28]. Moreover, stem cells secrete neurotrophic factors to modulate neuroplasticity and neurogenesis [29,30].

ESCs are self-renewing, totipotent cells that can differentiate into neuron progenitor cells (NPCs) in vitro, and this can result in a therapeutic effect when these cells are transplanted into $\mathrm{AD}$ animal models. ESC-derived NPCs were transplanted into an A $\beta$-injured rat model and the escape latency was significantly increased compared to phosphate buffered saline (PBS)-treated controls 2 weeks after the $\mathrm{A} \beta$ injection. The Morris water maze test was performed 16 weeks after transplantation, at which time the escape latency was found to have significantly decreased when compared to sham controls. Moreover, ESC-derived NPCs have been reported to be able to differentiate into astrocytic and neuron-like cells in vivo. These results suggested that ESC-derived NPCs ameliorated memory impairment. Although 
ESCs result in teratoma formation, it has been shown that ESC-derived NPCs can treat neurodegenerative diseases [31].

Human iPSCs were derived from skin cells by retroviral expression of octamer-binding transcription factor 4 (OCT4), sex determining region Y-box 2 (SOX2), proto-oncogene proteins c myc (cMYC), and kruppel-like factor 4 (KLF4), and can differentiate into neural cells. One report showed that induced iPSCs were generated from fibroblasts of familial ADs, and that these iPSCs differentiated into neurons that may increase $A \beta 42$ secretion. Moreover, $A \beta$ secretion from these differentiated neurons was affected by $\gamma$-secretase inhibitors, suggesting that these neurons have a pharmacological response to $\gamma$-secretase inhibitors. Thus these iPSCs may provide a potential strategy for the development of drugs against $\mathrm{AD}[32]$.

Mesenchymal stem cells (MSCs) are multi-potent progenitor cells that are mainly isolated from BM [33], adipose tissue [34], and the umbilical cord [35]. MSCs have been shown to differentiate into osteoblasts, adipocytes, and pancreatic islets [36,37]. BMMSCs have been reported to have the ability to modify and influence almost all the cells of the innate and adaptive immune systems mediated by BMMSC-soluble factors, including IL-6, IL-10, TGF- $\beta$, and PGE 2 [38-40]. BMMSCs strongly inhibited the maturation and functioning of monocyte-derived DCs by interfering selectively with the generation of immature cells via the inhibitory mediator of MSC-derived PGE2. BMMSCs have also been shown to alter the natural killer (NK) cell phenotype, and suppress the proliferation and cytokine secretion of NK cells $[41,42]$.

BMMSCs were able to home in on the injured brain and increase the number of positive cells for choline acetyltransferase. Furthermore, BMMSCs were able to remove A $\beta$ plaques from the hippocampus and to reduce $A \beta$ deposits through the activation of endogenous microglia in an induced $A D$ mouse model $[43,44]$. Moreover, human MSCs enhance autophagy, promote A $\beta$ clearance and increase neuronal survival in an A $\beta$-treated mouse model [45]. The transplantation of human MSCs has reduced infarct size and improved functional outcome, and autologous BMMSCs have been transfused to brain ischemic disease patients in clinical application [46]. In contrast, adipose-derived stem cells (ADSCs) were isolated from the inguinal fat pads of rats, and induced to differentiate into neurons or astrocyte-like cells in vitro. The transplantation of ADSCs allowed them to differentiate into neuron-like and astrocyte-like cells around the hematoma, accompanied with up-regulation of vascular endothelial growth factor expression and improvement of neural function, suggesting that ADSCs benefit neural differentiation and induce functional improvement in the rat [47]. When human ADSCs were intravenously injected into an AD mouse models, these cells could be found in the brain up to 12 days after their injection [48]. One report suggested that, when transplanted into the brain, adipose-derived MSCs (AMSCs) improved Ach levels as well as cognitive and locomotor functions in aged mice, and modulated microglia activation [29,49]. Human cord blood-derived stem cells have been shown to improve neuropathological and behavioral recovery from acute spinal cord trauma [50]. Human umbilical cord-derived MSCs can be induced to differentiate into neuron-like cells, and these cells were transplanted into an amyloid- $\beta$ precursor protein (A $\beta P P)$ and PS1 (A $\beta P P / P S 1)$ transgenic AD mouse model. The cognitive function was improved and A $\beta$ deposition was reduced after transplantation. These beneficial effects were associated with the activation of M2-like microglia [51,52]. 


\section{Intra-Bone Marrow-Bone Marrow Transplantation (IBM-BMT) and AD Mouse Model}

The MRL-MpJ-lpr/lpr (MRL/lpr) mouse is a mouse model for autoimmune diseases, and this strain shows abnormal radioresistant stem cells [53]. We have reported that BMT plus bone grafts can prevent the recurrence of autoimmune diseases, though this method failed in MRL/lpr mice because these mice are more radiosensitive after the onset of lupus nephritis [54]. Moreover, we reported that BMMSCs can become trapped in the liver and lung when BM cells are injected intravenously. We thus injected whole $\mathrm{BM}$ cells directly into the bone marrow cavity, as in IBM-BMT, for the treatment of autoimmune diseases in MRL/lpr mice. IBM-BMT, which not only replaces hematopoietic stem cells but also MSCs, has been proven to be the best method for allogeneic BMT because hematopoietic recovery is rapid, since the stroma cells directly home to the bone cavity and the restoration of $\mathrm{T}$ cell functions is complete even in donor-recipient combinations across the MHC barriers [55]. Up to this point, we have used IBM-BMT to successfully treat autoimmune diseases, osteoporosis, and Alzheimer's disease [56-58].

\subsection{IBM-BMT and Senescence-Accelerated Mouse Prone 8 (SAMP8)}

The senescence-accelerated mouse (SAM) was established via the selective inbreeding of the AKR/J strain of mice by Takeda et al. at Kyoto University. According to a graded score for senescence, life span, and pathologic phenotype, there are nine SAM-prone (SAMP) strains including SAMP1-3 and SAMP6-11, and three SAM-resistant strains (SAMR) including SAMR1, 4, and 5. These mice show age-related deficits in learning and memory and impaired immune response. Moreover, oxidative stress has been shown to be associated with mitochondrial dysfunction, and induced the excessive production of ROS and neurodegeneration in these two strains [59]. Heme oxygenase (HO) is an enzyme that catalyzes the degradation of heme. There are three isoforms: HO-1, HO-2 and HO-3. HO-1 is an inducible isoform, in responding to stress such as oxidative stress, hypoxia, and heavy metals. HO-2 is a constitutive isoform that is expressed under homeostatic conditions. HO-1 was reported to respond to excessive amyloid provocation and mitochondrial insufficiency, presenting chronic over-expression in the AD brain [60]. The SAMP8 was first reported to have age-related occurrence of deficits in learning and memory in 1986. SAMP8 mice showed significant impairment in the passive avoidance response, active avoidance tasks and spatial learning tasks when compared with age-matched SAMR1 [61]. Neuropathological studies showed that $A \beta$ deposition was similar to that in $A D$ in humans. $A \beta$ levels in the hippocampi of SAMP8 increased with aging, as shown by the enzyme-linked immuosorbent assay method, although no senile plaque-like structures were found. However, A $\beta$-like deposition increased with aging, as was confirmed by immunoblot analysis. Some Purkinje cells seemed to disappear with aging in the medial cerebellum and vermis of SAMP8, which was consistent with the finding in AD brains. Thus the SAMP8 is shown to be an acceptable rodent model for AD [62-65]. Moreover, A $\beta$ has been shown to play a central role in the pathophysiology of $A D$ through the induction of oxidative stress. One report has demonstrated that antisense oligonucleotides directed against PS-1 in old SAMP8 mice improved learning and memory deficits and reduced $A \beta$-mediated oxidative stress [66]. Another report indicated that hydrocotyle sibthorpioides administration prevented declines in spatial learning and memory by the scavenging of free radicals, up-regulating the activity of antioxidant enzymes, decreasing the level of $A \beta$ and ameliorating dysfunction in synaptic plasticity in SAMP8 mice [67]. 
$\mathrm{BM}$ cells have been shown to increase the number of activated microglia, and to reduce amyloid deposits via phagocytosis of $A \beta$ and thereby prevent the progression of $A D$ [68]. We used allogenic IBM-BMT to transfer normal BM stem cells in SAMP8. The 4-month-old SAMP8 mice received fractionated irradiation twice a day ( $4.5 \mathrm{~Gy} \times 2$, with a $4 \mathrm{~h}$ interval). One day after the irradiation, whole bone marrow cells from 8 -week-old C57BL/6 mice were injected into the recipient mice. These mice were then tested by the Morris water maze test, which is thought to be a sensitive assay for brain abnormalities, especially in the hippocampus [69]. We used it for examining the effects of IBM-BMT on spatial learning and memory ability. Three months after IBM-BMT, there was no significant difference between the escape latency and the swim speed of 4- and 7-month-old SAMR1. However, the escape latency of SAMP8 treated with IBM-BMT was significantly shorter than that of age-matched SAMP8 (Figure 1), although there was no difference in swim speed. The swim paths of SAMP8 treated with IBM-BMT were more directly toward the hidden platform than those of SAMP8. Analyses of the water maze tests showed the impairment of spatial memory in SAMP8 to have been ameliorated.

Figure 1. Escape latency of Senescence-Accelerated Mouse Prone 8 (SAMP8). (a) Untreated SAMP8; (b) SAMP8 treated with intra-bone marrow-bone marrow transplantation (IBM-BMT); and (c) SAM-resistant strains 1 (SAMR1).
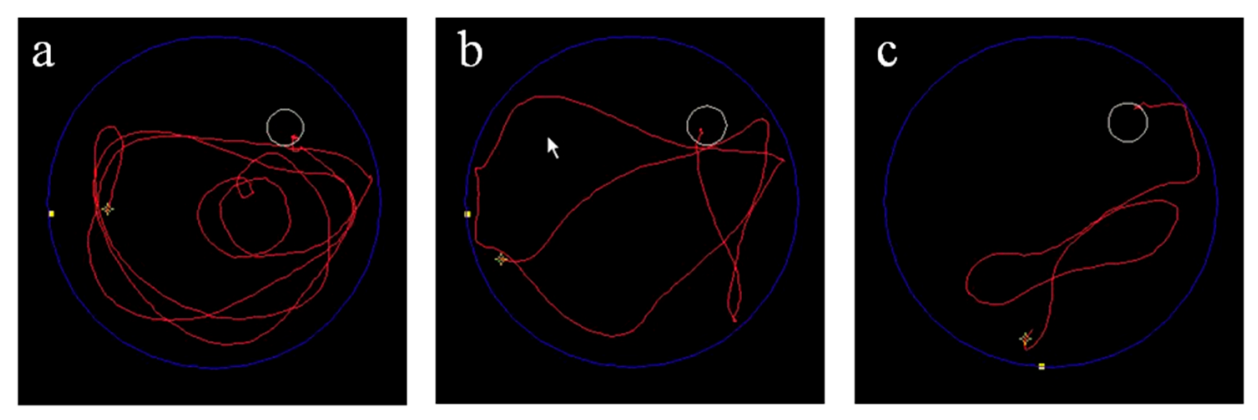

Proinflammatory cytokines are involved in the formation of neuritic plaques in Alzheimer's disease (AD) $[70,71]$. RT-PCR results showed that the expression of IL-6, IL-1 $\beta$ and inducible nitric oxide synthase (iNOS) decreased, while TGF- $\beta$ increased in the SAMP8 treated with IBM-BMT, compared to age-matched SAMP8. Western blot analysis to examine HO-1 expression showed a significant decrease in the ratio of HO-1 to actin in the SAMP 8 treated with IBM-BMT, compared to age-matched SAMP8 [58]. Our results suggested that IBM-BMT improved inflammation and oxidative stress in AD model mice.

\subsection{IBM-BMT and SAMP10}

SAMP10 show age-related deficits in learning and memory as well as emotional disorders, brain atrophy, shrinkage and loss of cortical neurons, accumulation of neuronal DNA damage, reduced hippocampal cholinergic receptors, decreased neurotrophic factors, increased sphingomyelinase, and elevated oxidative-nitrative stress. SAMP10 also showed decreased catecholamine synthesis in the cerebral cortex, related with the decline in learning and memory abilities with aging, and SAMP 10 have thus also been extensively used in studies as AD model mice [72,73]. In contrast, the microglia showed a shorter combined projection length, and there were fewer segments and tips in the SAMP10 than in the SAMR1, which is consistent with neuronal degeneration in the SAMP10. Moreover, the levels of 
age-related pro-inflammatory cytokines such as IL-1 $\beta$ and IFN- $\gamma$ showed greater increases in the SAMP10 than SAMR1 [74].

The immune system modulates CNS function and behavioral process. The thymus is a source of T cells, affecting the adaptive immunity. The immune system plays a central role in modulating learning, memory and neural plasticity. Under normal quiescent conditions, immune mechanisms are activated by environmental/psychological stimuli and positively regulate the remodeling of neural circuits, promoting memory consolidation, hippocampal long-term potentiation and neurogenesis. These beneficial effects of the immune system are mediated by complex interactions among brain cells with immune functions (particularly microglia and astrocytes), peripheral immune cells (particularly T cells and macrophages), neurons, and neural precursor cells [75]. We investigated whether neurodegenerative diseases were associated with thymus dysfunction in SAMP10. We first analyzed the lymphocyte populations in the peripheral blood by fluorescence-activated cell sorting (FACS). Our results showed that the percentages of CD4 and B220-positive cells were significantly lower, but the percentages of CD11b/Gr-1 double-positive cells were significantly higher in the 24-week-old SAMP10 than in the 6-week-old SAMP10, although there was no significant difference in the percentage of CD8 positive cells between 6-week- and 24-week-old SAMP10. Moreover, the percentage of CD4/TNF $\alpha$ T cells in the spleen of 24-week-old SAMP10 was significantly reduced compared to that of 6-week-old SAMP10, suggesting that the immune system was damaged in the SAMP10 [76].

Normal aging is associated with anatomical and functional changes in both the thymus and bone marrow. The aged thymus shows a disproportionate loss of thymic epithelial cells (TECs) and disrupted thymic architecture [77,78], resulting in an increased risk of autoimmunity through the escape of potentially self-reactive T cells from the disrupted thymic microenvironment. Sirt1 is a class III histone deacetylase within the sirtuin family of related proteins that is uniquely dependent on nicotinamide adenine dinucleotide ${ }^{+}\left(\mathrm{NAD}^{+}\right)$for catalysis. Sirt1 has been implicated in processes as varied as metabolism, differentiation, cancer, stress response and aging [79]. Sirt1 negatively regulates $\mathrm{T}$ cell activation and plays a major role in clonal $\mathrm{T}$ cell anergy in mice. Loss of Sirt1 function results in abnormally increased $\mathrm{T}$ cell activation and a breakdown of $\mathrm{CD} 4^{+} \mathrm{T}$ cell tolerance [80].

Our results showed that the thymus was significantly lighter and the percentage of $\mathrm{CD} 4^{+} \mathrm{CD} 8^{+}$was lower in the 24-week-old SAMP10 than the 6-week-old SAMP10. The expression of keratinocyte growth factor (KGF), Aire and Sirt1 was decreased on the TECs of 24-week-old SAMP10. Our previous report suggested that bone marrow cells contain the precursors of functional TECs, and that they can differentiate into TECs, thereby correcting thymic function [81]. RT-PCR showed that downregulated KGF, Aire and Sirt1 on the TEC of 24-week-old SAMP10 were improved after IBM-BMT. Thus, the findings showed that the dysfunction on the TEC of 24-week-old SAMP10 was modulated by allogeneic bone marrow cells in this experiment, suggesting that BMT might improve TEC function in SAMP10 [76]. We will focus future studies on the affects of stem cell transplantation on the pathologic changes in brain. Another report examined the finding that IBM-BMT facilitates the entry of transplanted BM cells into the brain parenchyma. IBM-BMT may thus prove beneficial in the experimental treatment of psychiatric and neurological diseases [82]. 


\section{Conclusions}

Aging is the greatest risk factor for the onset of AD. For the most part, pharmacological interventions are aimed at relieving the symptoms of $\mathrm{AD}$, but stem cell therapy not only has the potential to generate new neurons and replace damaged neurons but also to modulate the immune system (Figure 2).

Figure 2. Summary of Stem Cell Therapy for AD.

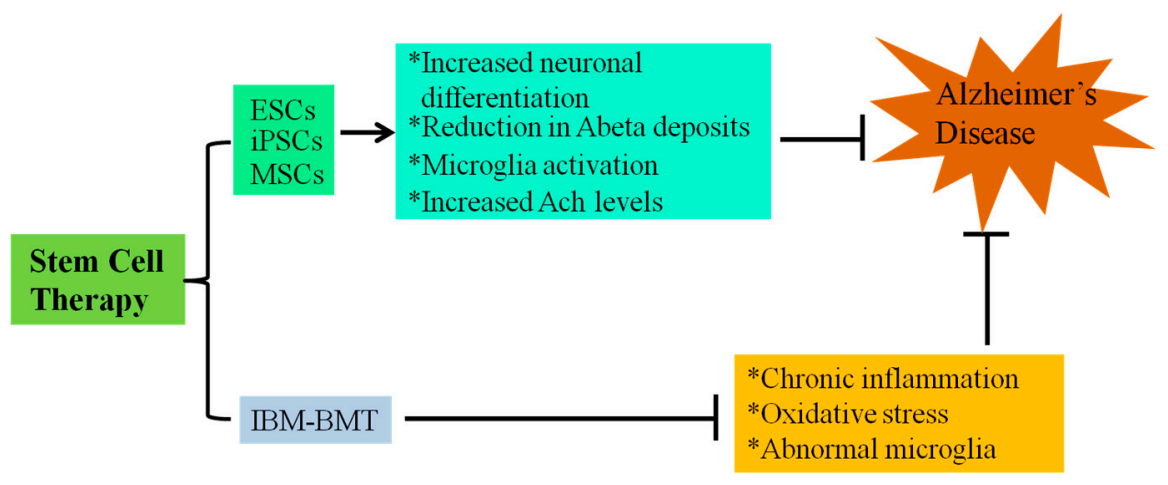

With further clarification of the mechanisms by which AD progresses, stem cell therapies may well prove to be both safe and effective treatments. In time, more advanced stem cell therapies hold the potential for the clinical treatment of this debilitating disease.

\section{Acknowledgments}

We would like to thank Hilary Eastwick-Field and Keiko Ando for their help in the preparation of the manuscript.

\section{Author Contributions}

Ming Li, Kequan Guo and Susumu Ikehara wrote the manuscript together.

\section{Conflicts of Interest}

The authors declare no conflict of interest.

\section{References}

1. Selkoe, D.J. Alzheimer's disease: Genes, proteins, and therapy. Physiol. Rev. 2001, 81, 741-766.

2. Persson, T.; Popescu, B.O.; Cedazo-Minguez, A. Oxidative stress in Alzheimer's disease: Why did antioxidant therapy fail? Oxid. Med. Cell Longev. 2014, 2014, 427318.

3. Demuro, A.; Smith, M.; Parker, I. Single-channel $\mathrm{Ca}^{2+}$ imaging implicates A $\beta$ 1-42 amyloid pores in Alzheimer's disease pathology. J. Cell Biol. 2011, 195, 515-524.

4. Kuhla, B.; Haase, C.; Flach, K.; Luth, H.J.; Arendt, T.; Munch, G. Effect of pseudophosphorylation and cross-linking by lipid peroxidation and advanced glycation end product precursors on tau aggregation and filament formation. J. Biol. Chem. 2007, 282, 6984-6991.

5. Eikelenboom, P.; Bate, C.; van Gool, W.A.; Hoozemans, J.J.; Rozemuller, J.M.; Veerhuis, R.; Williams, A. Neuroinflammation in Alzheimer's disease and prion disease. Glia 2002, 40, 232-239. 
6. Wenk, G.L. Neuropathologic changes in Alzheimer's disease. J. Clin. Psychiatry 2003, 64, 7-10.

7. Patel, A.N.; Jhamandas, J.H. Neuronal receptors as targets for the action of amyloid- $\beta$ protein (A $\beta$ ) in the brain. Expert Rev. Mol. Med. 2012, 14, e2.

8. Iqbal, K.; Alonso, A.C.; Gong, C.X.; Khatoon, S.; Pei, J.J.; Wang, J.Z.; Grundke-Iqbal, I. Mechanisms of neurofibrillary degeneration and the formation of neurofibrillary tangles. J. Neural Transm. Suppl. 1998, 53, 169-180.

9. Roberson, E.D.; Scearce-Levie, K.; Palop, J.J.; Yan, F.; Cheng, I.H.; Wu, T.; Gerstein, H.; Yu, G.Q.; Mucke, L. Reducing endogenous tau ameliorates amyloid $\beta$-induced deficits in an Alzheimer's disease mouse model. Science 2007, 316, 750-754.

10. Millington, C.; Sonego, S.; Karunaweera, N.; Rangel, A.; Aldrich-Wright, J.R.; Campbell, I.L.; Gyengesi, E.; Munch, G. Chronic neuroinflammation in Alzheimer's disease: New perspectives on animal models and promising candidate drugs. Biomed. Res. Int. 2014, 2014, 309129.

11. Munch, G.; Schinzel, R.; Loske, C.; Wong, A.; Durany, N.; Li, J.J.; Vlassara, H.; Smith, M.A.; Perry, G.; Riederer, P. Alzheimer's disease-Synergistic effects of glucose deficit, oxidative stress and advanced glycation endproducts. J. Neural Transm. 1998, 105, 439-461.

12. Loane, D.J.; Byrnes, K.R. Role of microglia in neurotrauma. Neurotherapeutics 2010, 7, 366-377.

13. Vilhardt, F. Microglia: Phagocyte and glia cell. Int. J. Biochem. Cell Biol. 2005, 37, 17-21.

14. Baune, B.T.; Ponath, G.; Rothermundt, M.; Roesler, A.; Berger, K. Association between cytokines and cerebral MRI changes in the aging brain. J. Geriatr. Psychiatry Neurol. 2009, 22, 23-34.

15. Velayutham, M.; Hemann, C.; Zweier, J.L. Removal of $\mathrm{H}_{2} \mathrm{O}_{2}$ and generation of superoxide radical: Role of cytochrome c and NADH. Free Radic. Biol. Med. 2011, 51, 160-170.

16. Jones, D.P. Radical-free biology of oxidative stress. Am. J. Physiol. Cell Physiol. 2008, 295, C849-C868.

17. Federico, A.; Cardaioli, E.; da Pozzo, P.; Formichi, P.; Gallus, G.N.; Radi, E. Mitochondria, oxidative stress and neurodegeneration. J. Neurol. Sci. 2012, 322, 254-262.

18. Opazo, C.; Huang, X.; Cherny, R.A.; Moir, R.D.; Roher, A.E.; White, A.R.; Cappai, R.; Masters, C.L.; Tanzi, R.E.; Inestrosa, N.C.; et al. Metalloenzyme-like activity of Alzheimer's disease $\beta$-amyloid. $\mathrm{Cu}$-dependent catalytic conversion of dopamine, cholesterol, and biological reducing agents to neurotoxic $\mathrm{H}_{2} \mathrm{O}_{2}$. J. Biol. Chem. 2002, 277, 40302-40308.

19. Meraz-Rios, M.A.; Toral-Rios, D.; Franco-Bocanegra, D.; Villeda-Hernandez, J.; Campos-Pena, V. Inflammatory process in Alzheimer's disease. Front. Integr. Neurosci. 2013, 7, 59.

20. Cha, M.Y.; Han, S.H.; Son, S.M.; Hong, H.S.; Choi, Y.J.; Byun, J.; Mook-Jung, I. Mitochondria-specific accumulation of amyloid $\beta$ induces mitochondrial dysfunction leading to apoptotic cell death. PLoS One 2012, 7, e34929.

21. Chapman, H.A.; Riese, R.J.; Shi, G.P. Emerging roles for cysteine proteases in human biology. Annu. Rev. Physiol. 1997, 59, 63-88.

22. Hook, V.; Toneff, T.; Bogyo, M.; Greenbaum, D.; Medzihradszky, K.F.; Neveu, J.; Lane, W.; Hook, G.; Reisine, T. Inhibition of cathepsin B reduces $\beta$-amyloid production in regulated secretory vesicles of neuronal chromaffin cells: Evidence for cathepsin B as a candidate $\beta$-secretase of Alzheimer's disease. Biol. Chem. 2005, 386, 931-940.

23. Mueller-Steiner, S.; Zhou, Y.; Arai, H.; Roberson, E.D.; Sun, B.; Chen, J.; Wang, X.; Yu, G.; Esposito, L.; Mucke, L.; Gan, L. Antiamyloidogenic and neuroprotective functions of cathepsin B: Implications for Alzheimer's disease. Neuron 2006, 51, 703-714. 
24. Marr, R.A.; Rockenstein, E.; Mukherjee, A.; Kindy, M.S.; Hersh, L.B.; Gage, F.H.; Verma, I.M.; Masliah, E. Neprilysin gene transfer reduces human amyloid pathology in transgenic mice. J. Neurosci. 2003, 23, 1992-1996.

25. Massoud, F.; Leger, G.C. Pharmacological treatment of Alzheimer disease. Can. J. Psychiatry 2011, $56,579-588$.

26. Mangialasche, F.; Kivipelto, M.; Mecocci, P.; Rizzuto, D.; Palmer, K.; Winblad, B.; Fratiglioni, L. High plasma levels of vitamin $\mathrm{E}$ forms and reduced Alzheimer's disease risk in advanced age. J. Alzheimers Dis. 2010, 20, 1029-1037.

27. Liu, Y.; Weick, J.P.; Liu, H.; Krencik, R.; Zhang, X.; Ma, L.; Zhou, G.M.; Ayala, M.; Zhang, S.C. Medial ganglionic eminence-like cells derived from human embryonic stem cells correct learning and memory deficits. Nat. Biotechnol. 2013, 31, 440-447.

28. Park, D.; Yang, Y.H.; Bae, D.K.; Lee, S.H.; Yang, G.; Kyung, J.; Kim, D.; Choi, E.K.; Lee, S.W.; Kim, G.H.; et al. Improvement of cognitive function and physical activity of aging mice by human neural stem cells over-expressing choline acetyltransferase. Neurobiol. Aging 2013, 34, 2639-2646.

29. Park, D.; Yang, G.; Bae, D.K.; Lee, S.H.; Yang, Y.H.; Kyung, J.; Kim, D.; Choi, E.K.; Choi, K.C.; Kim, S.U.; et al. Human adipose tissue-derived mesenchymal stem cells improve cognitive function and physical activity in ageing mice. J. Neurosci. Res. 2013, 91, 660-670.

30. Enciu, A.M.; Nicolescu, M.I.; Manole, C.G.; Muresanu, D.F.; Popescu, L.M.; Popescu, B.O. Neuroregeneration in neurodegenerative disorders. BMC Neurol. 2011, 11, 75.

31. Tang, J.; Xu, H.; Fan, X.; Li, D.; Rancourt, D.; Zhou, G.; Li, Z.; Yang, L. Embryonic stem cell-derived neural precursor cells improve memory dysfunction in $\mathrm{A} \beta(1-40)$ injured rats. Neurosci. Res. 2008, $62,86-96$.

32. Yagi, T.; Ito, D.; Okada, Y.; Akamatsu, W.; Nihei, Y.; Yoshizaki, T.; Yamanaka, S.; Okano, H.; Suzuki, N. Modeling familial Alzheimer's disease with induced pluripotent stem cells. Hum. Mol. Genet. 2011, 20, 4530-4539.

33. Campagnoli, C.; Roberts, I.A.; Kumar, S.; Bennett, P.R.; Bellantuono, I.; Fisk, N.M. Identification of mesenchymal stem/progenitor cells in human first-trimester fetal blood, liver, and bone marrow. Blood 2001, 98, 2396-2402.

34. Zuk, P.A.; Zhu, M.; Mizuno, H.; Huang, J.; Futrell, J.W.; Katz, A.J.; Benhaim, P.; Lorenz, H.P.; Hedrick, M.H. Multilineage cells from human adipose tissue: Implications for cell-based therapies. Tissue Eng. 2001, 7, 211-228.

35. Erices, A.; Conget, P.; Minguell, J.J. Mesenchymal progenitor cells in human umbilical cord blood. Br. J. Haematol. 2000, 109, 235-242.

36. Dominici, M.; le Blanc, K.; Mueller, I.; Slaper-Cortenbach, I.; Marini, F.; Krause, D.; Deans, R.; Keating, A.; Prockop, D.; Horwitz, E. Minimal criteria for defining multipotent mesenchymal stromal cells. The International Society for Cellular Therapy position statement. Cytotherapy 2006, 8, 315-317.

37. Tang, D.Q.; Cao, L.Z.; Burkhardt, B.R.; Xia, C.Q.; Litherland, S.A.; Atkinson, M.A.; Yang, L.J. In vivo and in vitro characterization of insulin-producing cells obtained from murine bone marrow. Diabetes 2004, 53, 1721-1732.

38. Beyth, S.; Borovsky, Z.; Mevorach, D.; Liebergall, M.; Gazit, Z.; Aslan, H.; Galun, E.; Rachmilewitz, J. Human mesenchymal stem cells alter antigen-presenting cell maturation and induce T-cell unresponsiveness. Blood 2005, 105, 2214-2219. 
39. Ramasamy, R.; Fazekasova, H.; Lam, E.W.; Soeiro, I.; Lombardi, G.; Dazzi, F. Mesenchymal stem cells inhibit dendritic cell differentiation and function by preventing entry into the cell cycle. Transplantation 2007, 83, 71-76.

40. Aggarwal, S.M.; Pittenger, F. Human mesenchymal stem cells modulate allogeneic immune cell responses. Blood 2005. 105, 1815-1822.

41. Sotiropoulou, P.A.; Perez, S.A.; Gritzapis, A.D.; Baxevanis, C.N.; Papamichail, M. Interactions between human mesenchymal stem cells and natural killer cells. Stem Cells 2006, 24, 74-85.

42. Ryan, J.M.; Barry, F.; Murphy, J.M.; Mahon, B.P. Interferon- $\gamma$ does not break, but promotes the immunosuppressive capacity of adult human mesenchymal stem cells. Clin. Exp. Immunol. 2007, 149, 353-363.

43. Salem, A.M.; Ahmed, H.H.; Atta, H.M.; Ghazy, M.A.; Aglan, H.A. Potential of bone marrow mesenchymal stem cells in management of Alzheimer's disease in female rats. Cell Biol. Int. 2014, doi:10.1002/cbin.10331.

44. Lee, J.K.; Jin, H.K.; Bae, J.S. Bone marrow-derived mesenchymal stem cells reduce brain amyloid- $\beta$ deposition and accelerate the activation of microglia in an acutely induced Alzheimer's disease mouse model. Neurosci. Lett. 2009, 450, 136-141.

45. Shin, J.Y.; Park, H.J.; Kim, H.N.; Oh, S.H.; Bae, J.S.; Ha, H.J.; Lee, P.H. Mesenchymal stem cells enhance autophagy and increase $\beta$-amyloid clearance in Alzheimer disease models. Autophagy 2014, 10, 32-44.

46. Honmou, O.; Houkin, K.; Matsunaga, T.; Niitsu, Y.; Ishiai, S.; Onodera, R.; Waxman, S.G.; Kocsis, J.D. Intravenous administration of auto serum-expanded autologous mesenchymal stem cells in stroke. Brain 2011, 134, 1790-1807.

47. Chen, J.; Tang, Y.X.; Liu, Y.M.; Hu, X.Q.; Liu, N.; Wang, S.X.; Zhang, Y.; Zeng, W.G.; Ni, H.J.; Zhao, B.; et al. Transplantation of adipose-derived stem cells is associated with neural differentiation and functional improvement in a rat model of intracerebral hemorrhage. CNS Neurosci. Ther. 2012, 18, 847-854.

48. Ha, S.; Ahn, S.; Kim, S.; Joo, Y.; Chong, Y.H.; Suh, Y.H.; Chang, K.A. In vivo imaging of human adipose-derived stem cells in Alzheimer's disease animal model. J. Biomed. Opt. 2014, 19, 051206.

49. Ma, T.; Gong, K.; Ao, Q.; Yan, Y.; Song, B.; Huang, H.; Zhang, X.; Gong, Y. Intracerebral transplantation of adipose-derived mesenchymal stem cells alternatively activates microglia and ameliorates neuropathological deficits in Alzheimer's disease mice. Cell Transplant. 2013, 22, S113-S126.

50. Schira, J.; Gasis, M.; Estrada, V.; Hendricks, M.; Schmitz, C.; Trapp, T.; Kruse, F.; Kogler, G.; Wernet, P.; Hartung, H.P.; et al. Significant clinical, neuropathological and behavioural recovery from acute spinal cord trauma by transplantation of a well-defined somatic stem cell from human umbilical cord blood. Brain 2012, 135, 431-446.

51. Darlington, D.; Deng, J.; Giunta, B.; Hou, H.; Sanberg, C.D.; Kuzmin-Nichols, N.; Zhou, H.D.; Mori, T.; Ehrhart, J.; Sanberg, P.R.; Tan, J. Multiple low-dose infusions of human umbilical cord blood cells improve cognitive impairments and reduce amyloid- $\beta$-associated neuropathology in Alzheimer mice. Stem Cells Dev. 2013, 22, 412-421.

52. Yang, H.; Xie, Z.; Wei, L.; Yang, S.; Zhu, Z.; Wang, P.; Zhao, C.; Bi, J. Human umbilical cord mesenchymal stem cell-derived neuron-like cells rescue memory deficits and reduce amyloid- $\beta$ deposition in an A $\beta$ PP/PS1 transgenic mouse model. Stem Cell Res. Ther. 2013, 4, 76. 
53. Ikehara, S.; Yasumizu, R.; Inaba, M.; Izui, S.; Hayakawa, K.; Sekita, K.; Toki, J.; Sugiura, K.; Iwai, H.; Nakamura, T.; et al. Long-term observations of autoimmune-prone mice treated for autoimmune disease by allogeneic bone marrow transplantation. Proc. Natl. Acad. Sci. USA 1989, 86, 3306-3310.

54. Ishida, T.; Inaba, M.; Hisha, H.; Sugiura, K.; Adachi, Y.; Nagata, N.; Ogawa, R.; Good, R.A.; Ikehara, S. Requirement of donor-derived stromal cells in the bone marrow for successful allogeneic bone marrow transplantation. Complete prevention of recurrence of autoimmune diseases in MRL/MP-Ipr/Ipr mice by transplantation of bone marrow plus bones (stromal cells) from the same donor. J. Immunol. 1994, 152, 3119-3127.

55. Kushida, T.; Inaba, M.; Hisha, H.; Ichioka, N.; Esumi, T.; Ogawa, R.; Iida, H.; Ikehara, S. Intra-bone marrow injection of allogeneic bone marrow cells: A powerful new strategy for treatment of intractable autoimmune diseases in MRL/lpr mice. Blood 2001, 97, 3292-3299.

56. Kushida, T.; Ueda, Y.; Umeda, M.; Oe, K.; Okamoto, N.; Iida, H.; Abraham, N.G.; Gershwin, M.E.; Ikehara, S. Allogeneic intra-bone marrow transplantation prevents rheumatoid arthritis in SKG/Jcl mice. J. Autoimmun. 2009, 32, 216-222.

57. Takada, K.; Inaba, M.; Ichioka, N.; Ueda, Y.; Taira, M.; Baba, S.; Mizokami, T.; Wang, X.; Hisha, H.; Iida, H.; et al. Treatment of senile osteoporosis in SAMP6 mice by intra-bone marrow injection of allogeneic bone marrow cells. Stem Cells 2006, 24, 399-405.

58. Li, M.; Inaba, M.; Guo, K.; Abraham, N.G.; Ikehara, S. Amelioration of cognitive ability in senescence-accelerated mouse prone 8 (SAMP8) by intra-bone marrow-bone marrow transplantation. Neurosci. Lett. 2009, 465, 36-40.

59. Chiba, Y.; Shimada, A.; Kumagai, N.; Yoshikawa, K.; Ishii, S.; Furukawa, A.; Takei, S.; Sakura, M.; Kawamura, N.; Hosokawa, M. The senescence-accelerated mouse (SAM): A higher oxidative stress and age-dependent degenerative diseases model. Neurochem. Res. 2009, 34, 679-687.

60. Schipper, H.M. Heme oxygenase-1: Role in brain aging and neurodegeneration. Exp. Gerontol. 2000, 35, 821-830.

61. Miyamoto, M.; Kiyota, Y.; Yamazaki, N.; Nagaoka, A.; Matsuo, T.; Nagawa, Y.; Takeda, T. Age-related changes in learning and memory in the senescence-accelerated mouse (SAM). Physiol. Behav. 1986, 38, 399-406.

62. Takemura, M.; Nakamura, S.; Akiguchi, I.; Ueno, M.; Oka, N.; Ishikawa, S.; Shimada, A.; Kimura, J.; Takeda, T. $\beta /$ A4 proteinlike immunoreactive granular structures in the brain of senescence-accelerated mouse. Am. J. Pathol. 1993, 142, 1887-1897.

63. Kumar, V.B.; Farr, S.A.; Flood, J.F.; Kamlesh, V.; Franko, M.; Banks, W.A.; Morley, J.E. Site-directed antisense oligonucleotide decreases the expression of amyloid precursor protein and reverses deficits in learning and memory in aged SAMP8 mice. Peptides 2000, 21, 1769-1775.

64. Flood, J.F.; Morley, J.E. Early onset of age-related impairment of aversive and appetitive learning in the SAMP8 mouse. J. Gerontol. 1992, 47, B52-B59.

65. Sjobeck, M.; Englund, E. Alzheimer's disease and the cerebellum: A morphologic study on neuronal and glial changes. Dement. Geriatr. Cogn. Disord. 2001, 12, 211-218.

66. Fiorini, A.; Sultana, R.; Forster, S.; Perluigi, M.; Cenini, G.; Cini, C.; Cai, J.; Klein, J.B.; Farr, S.A.; Niehoff, M.L.; et al. Allan butterfield. Antisense directed against PS-1 gene decreases brain oxidative markers in aged senescence accelerated mice (SAMP8) and reverses learning and memory impairment: A proteomics study. Free Radic. Biol. Med. 2013, 65, 1-14. 
67. Lin, X.; Huang, R.; Zhang, S.; Wei, L.; Zhuo, L.; Wu, X.; Tang, A.; Huang, Q. Beneficial effects of asiaticoside on cognitive deficits in senescence-accelerated mice. Fitoterapia 2013, 87, 69-77.

68. Simard, A.R.; Soulet, D.; Gowing, G.; Julien, J.P.; Rivest, S. Bone marrow-derived microglia play a critical role in restricting senile plaque formation in Alzheimer's disease. Neuron 2006, 49, 489-502.

69. Skelton, R.W.; McNamara, R.K. Bilateral knife cuts to the perforant path disrupt spatial learning in the Morris water maze. Hippocampus 1992, 2, 73-80.

70. Strauss, S.; Bauer, J.; Ganter, U.; Jonas, U.; Berger, M.; Volk, B. Detection of interleukin-6 and a 2-macroglobulin immunoreactivity in cortex and hippocampus of Alzheimer's disease patients. Lab. Investig. 1992, 66, 223-230.

71. Hosokawa, M. A higher oxidative status accelerates senescence and aggravates age-dependent disorders in SAMP strains of mice. Mech. Ageing Dev. 2002, 123, 1553-1561.

72. Takeda, T.; Matsushita, T.; Kurozumi, M.; Takemura, K.; Higuchi, K.; Hosokawa, M. Pathobiology of the senescence-accelerated mouse (SAM). Exp. Gerontol. 1997, 32, 117-127.

73. Shimada, A.; Hasegawa-Ishii, S. Senescence-accelerated mice (SAMs) as a model for brain aging and immunosenescence. Aging Dis. 2011, 2, 414-435.

74. Hasegawa-Ishii, S.; Takei, S.; Chiba, Y.; Furukawa, A.; Umegaki, H.; Iguchi, A.; Kawamura, N.; Yoshikawa, K.; Hosokawa, M.; Shimada, A. Morphological impairments in microglia precede age-related neuronal degeneration in senescence-accelerated mice. Neuropathology 2011, 31, 20-28.

75. Yirmiya, R.; Goshen, I. Immune modulation of learning, memory, neural plasticity and neurogenesis. Brain Behav. Immun. 2011, 25, 181-213.

76. Li, M.; Shi, M.; Abraham, N.G.; Ikehara, S. Improved expression of Sirt1 on thymic epithelial cells of SAMP10 after intra bone marrow-bone marrow transplantation. Cell Transplant. 2014, 23, 1019-1029.

77. Gray, D.H.; Seach, N.; Ueno, T.; Milton, M.K.; Liston, A.; Lew, A.M.; Goodnow, C.C.; Boyd, R.L. Developmental kinetics, turnover, and stimulatory capacity of thymic epithelial cells. Blood 2006, 108, 3777-3785.

78. Gui, J.; Zhu, X.; Dohkan, J.; Cheng, L.; Barnes, P.F.; Su, D.M. The aged thymus shows normal recruitment of lymphohematopoietic progenitors but has defects in thymic epithelial cells. Int. Immunol. 2007, 19, 1201-1211.

79. Calvanese, V.; Fraga, M.F. Sirt1 brings stemness closer to cancer and aging. Aging 2011, 3, 162-167.

80. Zhang, J.; Lee, S.M.; Shannon, S.; Gao, B.; Chen, W.; Chen, A.; Divekar, R.; McBurney, M.W.; Braley-Mullen, H.; Zaghouani, H.; et al. The type III histone deacetylase Sirt1 is essential for maintenance of T cell tolerance in mice. J. Clin. Investig. 2009, 119, 3048-3058.

81. Takaki, T.; Hosaka, N.; Miyake, T.; Cui,W.; Nishida, T.; Inaba, M.; Ikehara. S. Presence of donor-derived thymic epithelial cells in B6 $\rightarrow$ MRL/lpr mice after allogeneic intra-bone marrow-bone marrow transplantation (IBM-BMT). J. Autoimmun. 2008, 31, 408-415.

82. Hasegawa-Ishii, S.; Shimada, A.; Inaba, M.; Li, M.; Shi, M.; Kawamura, N.; Takei, S.; Chiba, Y.; Hosokawa, M.; Ikehara, S. Selective localization of bone marrow-derived ramified cells in the brain adjacent to the attachments of choroid plexus. Brain Behav. Immun. 2013, 29, 82-97.

(C) 2014 by the authors; licensee MDPI, Basel, Switzerland. This article is an open access article distributed under the terms and conditions of the Creative Commons Attribution license (http://creativecommons.org/licenses/by/4.0/). 